\title{
Photodetection of Micro-Biomotility and Ecological Monitoring of Lower Organisms
}

\author{
Svetlana B. Norina ${ }^{1}$, Alexander N. Shalygin ${ }^{1}$, Stanislav F. Rastopov ${ }^{2, *}$ \\ ${ }^{1}$ Faculty of Physics, Lomonosov Moscow State University, Leninskie Gory, Moscow 119991 Russia \\ ${ }^{2}$ Prokhorov General Physics Institute, Russian Academy of Sciences, Moscow, Russia \\ *Corresponding Author: sbnor@bk.ru
}

Copyright $@ 2013$ Horizon Research Publishing All rights reserved.

\begin{abstract}
A method is proposed for the ecological monitoring of lower organisms by using the frequency of mechanical vibrations, and the real time detection of their motion. The synchronous mechanical oscillations of invertebrates were detected under constant frequency bands. The waves generated by these organisms in their normal state were observed, as well as the vibrations splitting response under various external conditions such as: a magnetic field gradient and thermal stress. The spectroscopic analysis of the intensity fluctuations of light scattered by the organisms allowed the detection of complex biorhythms. Used optical portable spectrometer detected Fourier spectra and real-time rhythms of cilia beating and drifting motility. The common main cilia beating frequency was found between 16-17 Hz for Artemia Salina brine shrimps. The experimental results demonstrated that the reaction of cilia beating to a gradient of a magnetic field is weaker than the reaction to other stress induced by temperature gradients.
\end{abstract}

Keywords Spectroscopy of Intensity Fluctuations, Cilia Beating, Motility Photo-Detection, Artemia Salina Brine Shrimp, Ecological Monitoring.

\section{Introduction}

Lower organisms react to, and thus can detect the presence of chemicals in their living environment [1]. A study of the influence that the external medium has on biological systems is a very actual problem, particularly on the ciliary beating parameters, the swimming behavior, the contractile rhythms of motile organisms and tissues. The main function of cilia is to transport materials and to swim in water. Each cilium beats in a periodic manner, all the cilia are coordinated in time and space to create a wave. The movement of cilia is called metachronism, and its main functions are to transport food and other materials, and to swim in water. In this work, the possible mechanism is suggested for the influence that certain changes in the environment conditions may have on the ciliary wave direction and frequency [2]. A ciliary activity is primarily responsible for the transport of a feed and a foreign material. Such studies have given a valuable information on the response of the clearance rates to various stimuli such as drugs, the rate of hydration, various stress states [3]. Most of the information about the structure and function of cilia has been derived from studies of cilia from invertebrate organisms, algae Chlamidomonadas flagella and sperm flagella [4].

Ciliary motility was examined optically in tissue cultures from tracheal epithelium as a function of extracellular ATP (adenosine tri phosphate) of $10^{-7}-10^{-3} \mathrm{M}$ and the frequency of ciliary beating increased by a factor 3 from chemical changes of ciliary metabolism [5]. The laser, electro-magnetic fields, chemical compounds, toxical mixes, heavy metals cations, pollutant water shift and make diffused the normal Fourier spectrum which is usually reproducible in constant conditions.

Brine shrimps Artemia Salina are well-known ecological indicator, they live in very salty waters and they can even survive in dry state being in cysts under the embryonic stage of the development [6].

The motile speedy registration of swimming Protozoa cells was reported [7]. Inhibiting the half spermatozoa motility, the concentration and the acute toxicity was studied of certain chemical compounds in water such as: methanol, ethanol, methylmetacrylate, dichloroethane, and formaldehyde. Protozoa can change the motor behavior as the response on stimuli.

In our work, a method has been developed based on the photodetection of micro-biomotility, for the quantitative analysis and prognosis of the effect of environmental stress factors on motile lower organisms.

\section{Experimental Materials and Methods}

\subsection{Spectroscopic Method for Studying Fluctuations of The Scattered Light Intensity}

The portable device [8] placed into the ocular tube of microscopy (instead of the ocular) was used to measure 
visually targeted structures of specimen for their Fourier-spectra analysis and dynamical processes documentation in a spectral range from $1 \mathrm{~Hz}$ to $1000 \mathrm{~Hz}$ and permitted motility analysis under high spatial resolution of moving structures up to $1 \mu \mathrm{m}$. The fluctuations of the photoelectric current, which correspond to the cell beating, were amplified and converted with an analog-to-digital device to the LabView system, adjusted to document the intensity fluctuations, the real-time parameters, and the temperature measurements. Two photodiodes were components of the difference scheme detecting signal from intensity fluctuations. Software Lab View realized Fourier Transform of signals. The photo-detectors were placed at the optic microscopic image plane and the fluctuations of light intensity due to beating of object were used to study the ciliary and motile activity.

Microscopic images of the object were projected simultaneously onto the photodiode planes coinciding with the ocular observation plane of the microscope. The rhythmic events counted every $2 \mathrm{~s}$ were documented on a six graphs display containing real-time dependencies: frequency, temperature, amplitude, intensity via time, frequency via temperature, and Fourier-spectra.

\subsection{Organism Species and Sample Preparation}

Lower animal species, invertebrates: brine shrimps Artemia Salina were observed in light phase-contrast microscope Olympus BX-51 in clear water in hermetic glass cell $3 \cdot 15 \cdot 15 \mathrm{~mm}$ of sizes, supplemented with an injecting tube of $1 \mathrm{~mm}$ diameter and containing the cotton net or the magnet pieces to fix in several experiments the motile organisms in the place of the viewing. The photo-detector was placed at the optic microscopic image plane and the fluctuations of light intensity due to beating of object were used to study the ciliary and motile activity.

Shrimp eggs were incubated in $0.5 \% \mathrm{NaCl}$ solution for 3 days, and nauplii shrimps by sizes $90-100 \mu \mathrm{m}$ hatched from eggs.

Temperature was increased with heater up to 35 degrees to affect on objects as the stress factor.

\subsection{Analytical Method Based on the Magnetic Fixation Principle}

The permanent magnet was used to magnetize Ni-wire $[9,10]$ by $325 \mu \mathrm{m}$ in diameter. Artemia Salina shrimps attracted to the magnetized wire due to presence of ferritin super-paramagnetic macromolecules inside organisms, while Artemia Salina shrimp eggs were diamagnetic and repelled from gradient magnetic field. To measure the magnetic properties of single Artemia eggs and shrimps their drifts in a gradient magnetic field were recorded using CCD camera.

Magnetic susceptibility can be obtained from the equality of a magnetic ponder-motive force and drag force by Stokes:
$6 \pi v R_{\text {eff }} \vec{v}=1 / 2\left(\chi_{p}-\chi_{f}\right) \cdot V \cdot \nabla(\vec{H} \cdot \vec{H})$, where $\mathrm{H}_{0}$ is the applied permanent magnetic field by 1-10 kOe, $\chi_{\mathrm{p}}$ and $\chi_{\mathrm{f}}$ are the susceptibility of the particles and the fluid, $R_{\text {eff }}{ }^{-}$ effective radius of an object, $\eta$ is viscosity coefficient, $v$ is the velocity in a magnetic field. The gradient magnetic parameter value was $\mathbf{H} \cdot \operatorname{grad} \mathbf{H}=5 \cdot 10^{7} \mathrm{Oe}^{2} / \mathrm{cm}$, the magnetic force was nearly $10^{-7}$ dynes.

\subsection{Instruments}

The photosensitive elements were the photodiodes by the type QD7-5. The light phase-contrast microscope Olympus BX-51 was used with the portable device. The band pass filter is arranged to pass only signals in the frequency range between about $1 \mathrm{~Hz}$ and about $1000 \mathrm{~Hz}$ and the resulting signal supplied and digitised by the ADC (analog-to-digital converter) is a sensitive and responsive measure of the intensity fluctuations of scattering particles in the sample. The ADC comprised a standard PC (personal computer) sound card.

\section{Results and Discussion}

In Figures 1 and 2 the control graphs are presented. Under the placing the detector with a focus for the single Artemia shrimp animal the photo-electronic signal had characteristic shape at the cilia beating frequency as one can see on Figure 1 , where the three phases of the cilia beating are demonstrated for Artemia Salina brine shrimp: the effective, the recovery and the rest phases.

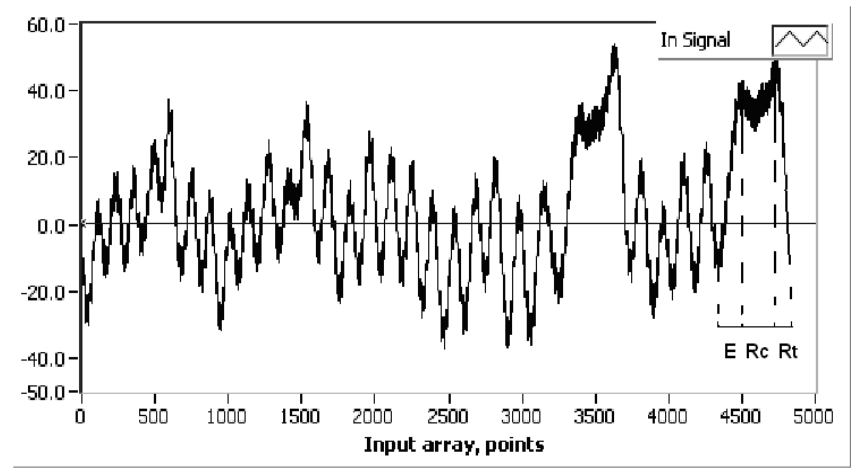

Figure 1. Typical plot of detected signal (Voltage) for the normal rhythms of Artemia Salina brine shrimp: the real-time graph is the metachronal rhythm from the single cilium of Artemis Salina. Horisontal axis - Time, 1000 points $=0,4 \mathrm{~s}$; vertical axis- Voltage, $\mathrm{mV}$. The various regions are indicated: E-effective, $R_{c}$ - recovery and $R_{t}$ - rest phases of beating cycle.

The duration of each phase on Figure 1 of the beat cycle varies according to the mean beating frequency and animal species. The quasi-periodic optical signal which describes ciliary motility is defined by three parameters: frequency, amplitude and phase. The first two parameters can be 
obtained with photoelectric devices. The third parameter, a phase, or degree of synchronization between beating cilia located at two different areas [2] was based on simultaneous measurements of scattered light from two points on the ciliary epithelium.

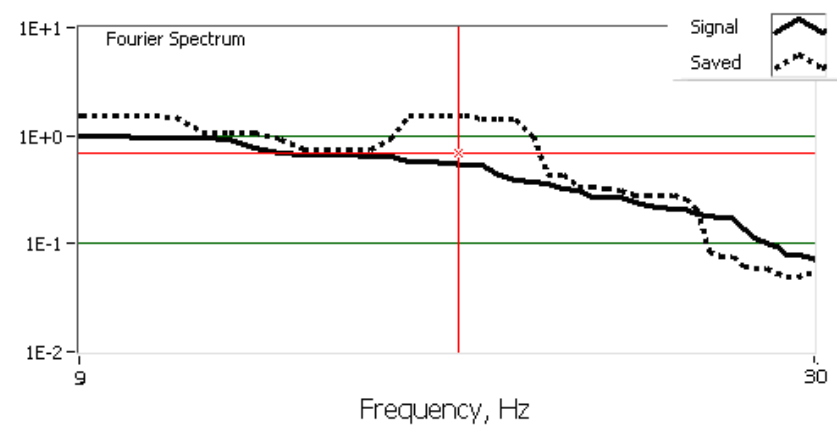

Figure 2. Typical Fourier spectrum of metachronal rhythms (dashed line) and control (solid line). Vertical axis - frequency harmonic amplitude, $\mathrm{mV}$. Horizontal axis - frequency, Hz. Fourier spectrum was performed for dependence of the signal on time presented in Figure 1.

The range of synchronization between cilia is of the order of one ciliary cell corresponding to a metachronal wave length. As beating frequency increased the duration of the effective phase and of the recovery stroke shortened while the duration of rest phase became shorter than the recovery stroke. The using of a small aperture allowed the recording of the signal from the single silium and the real-time images of beating cycle which is the sum of the three phases.

Artemia Salina brine shrimps revealed the characteristic Fourier-spectra and real-time oscillograms for the hatching and adult stages of the development. A frequency band $16.7 \pm 1.1(\mathrm{~Hz})$ being reproducible for nauplii remained the same for adult species broadening and shifting considerably under environmental influences.

The histograms in Figure 3 show data obtained under the statistical analysis of more than 200 shrimps in normal state and after stress influences. The active shrimps gave the narrow frequencies bands which were shifting and spreading under temperature increase and the long keeping time without meal-substrates. The homogeneity of Artemia Salina suspension with the frequency indication was revealed for the fresh normal brine shrimps that allowed the estimation of the physiological state of the population; the typical response of the frequency band broadening and shifting were revealed under the temperature increasing up to 35 degrees.

In Figure 4(a) the shrimp is attracted to the magnetized wire due to the natural internal ferritin, while shrimp eggs are repelled from the wire. Figure 4(b) demonstrates the narrow bands nearly 16.5, and frequency harmonics $33,49.5 \mathrm{~Hz}$ of Fourier-spectrum for Artemia Salina brine shrimp obtained in the spatial spot $150 \mu \mathrm{m}$ for the magnetically fixed single shrimp. The influence of a gradient magnetic field (the gradient magnetic value was $\mathrm{H} \cdot \operatorname{gradH}=5 \cdot 107 \mathrm{Oe} 2 / \mathrm{cm}$ ) on Fourier spectrum of Artemia Salina shrimp caused the appearance of new frequency bands (instead only $16.5 \mathrm{~Hz}$ band in control)
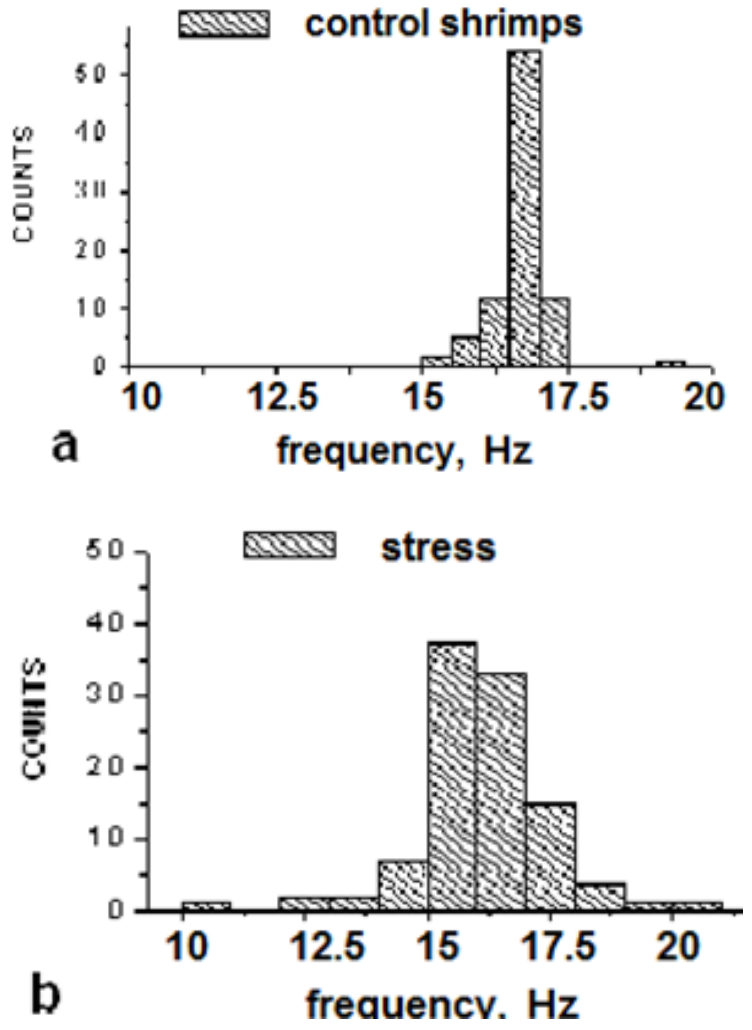

Figure 3. Histograms of the frequency beating of shrimps: control (a) and stressed (b).

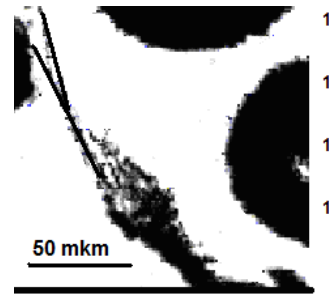

magnetized wire

a

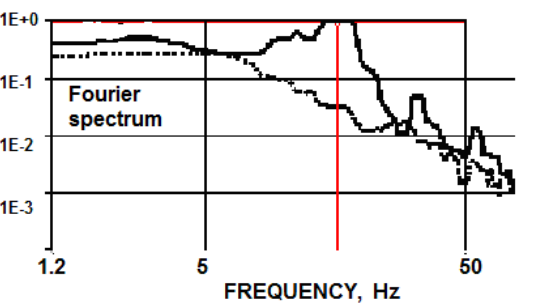

b
Figure 4. The influence of a gradient magnetic field on the Fourier spectrum of Artemia Salina shrimp fixated by a magnetic force: (a) is the shrimp near magnetized wire; (b) shows new frequency bands (instead only $16.5 \mathrm{~Hz}$ band in control) appearing in the result of magnetic fixation of a shrimp in the respect to water (dash line); vertical axis - frequency harmonic amplitude, $\mathrm{mV}$; horizontal axis - frequency beating of shrimp.

\section{Conclusion}

The quasi-periodic optical signal which describes ciliary motility of invertebrates brine shrimp Artemia Salina was defined at $16.5 \mathrm{~Hz}$ in the range of a synchronization. The frequency changes were established under the temperature and magnetic stress influences with respect to control organisms. The method gives the conclusions about the state of metachronal coordination of cilia if the characteristic Fourier-bands are narrow and the conclusion about the disturbance in the synchronization if the bands are splitting, broadening and shifting as it usually occurs under the stress 
influences effecting on the oscillating populations. The light microscopic dynamical processes could be convenient assays for the ecological monitoring.

\section{REFERENCES}

[1] Abernethy S, Bobra A, Shiu W, Wells P, Mackay D. Acute lethal toxicity of hydrocarbons and chlorinated hydrocarbons to two planktonic crustaceans: the key role of organism-water partitioning. Aquat Toxicol, 1986; 8(3):163-174.

[2] Gheber L, Priel Z. On metachronism in ciliary systems; a model describing the dependence of the metachronal wave properties on the intrinsic ciliary parameters. Cell Mot Cyt , 1990;16:167-181.

[3] Silverberg A. Functional activity of ciliated outgrowths from cultured human nasal and tracheal epithelia. Biorheology, 1990;27: 559-565.

[4] Holwill E, Foster G. Biophysical aspects and modelling of cilliary motility. Cell Mot Cyt, 1995;32:114-120.
[5] Ovadyahy D, Eshel D, Priel Z. Intensification of ciliary motility by extra-cellular ATP. Biorheology, 1988; 25:489-501.

[6] Barahona M, Sanchez-Fortun S. Toxicity of carbamates to the brine shrimp Artemia salina and the effect of atropine, BW284c51, iso-OMPA and 2-PAM on carbaryl toxicity. Environment Pollution, 1999;104(3):469-476.

[7] R. Kayumov, A. Yeskov. The toxicological estimation of individual chemical compounds and complex mixures by means the motile cellular cell tested objects, Bul Exp Biol Med, 1988;105:48-50 (in Russian).

[8] Rastopov S. Optical detection of particles in a liquid medium. United States Patent Applicatin 20040070756 A1

[9] Norina S, Park SH, Kim J, Cho S, Shalygin A, Soh KS. Image analysis of bioparticles accumulation and diamagnetic alignment in high gradient magnetic field. J.Biomedical Optics, 2005;10/5: 051702-1 - 051702-12.

[10] Norina S, Kim J, Soh KS. Gradient magnetic field effects on separation, Fourier-spectra and motion of chromatin particles and cells, International Journal of Applied Electromagnetics and Mechanics, 2007; 25:419-427.. 